\title{
Monopoles and deconfinement transition in finite temperature $S U(2)$ QCD
}

\author{
Shun-ichi Kitahara ${ }^{\mathrm{a}}$, Yoshimi Matsubara ${ }^{\mathrm{b}}$ and Tsuneo Suzuki \\ a Department of Physics, Kanazawa University, Kanazawa 920-11, Japan,

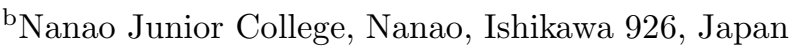

We investigate the role of monopoles in the deconfinement transition of finite temperature $S U(2)$ QCD in the maximally abelian gauge. In the confinement phase a long monopole loop exists in each configuration, whereas no long loop exists in the deep deconfinement region. Balancing of the energy and the entropy of loops of the maximally extended monopoles can explain the occurrence of the deconfinement transition.

\section{Introduction}

Recent studies in abelian projected QCD on lattices show that condensation of monopoles seems to cause confinement of quarks [1]. The 'tHooft's idea of abelian projection is to fix the gauge in such a way that the maximal torus group remains unbroken. QCD is regarded as an abelian theory with monopoles [2]. If the monopoles make Bose condensation, quarks are confined due to the dual Meissner effect.

The long range property is expected to be important in the confinement phase of QCD. Considering extended monopoles which corresponds to performing block spin transformations on the dual lattice is important [3]. Recently interesting results have been found [it:

1. A monopole effective action can be calculated for extended monopoles.

2. As the extendedness of monopoles becomes bigger, $\beta_{c}$ tends to be larger. Here $\beta_{c}$ is where balancing of the energy and the entropy of a monopole loop occurs.

3. The effective action for $n^{3}$ extended monopoles becomes the same for any $n$ written in terms of $b, b=n a(\beta)$. Hence corresponding to $\beta_{c}$, there exists a unique value $b_{c}=5.2 \times 10^{-3} \Lambda_{L}^{-1}$ for extended monopoles, above which monopoles condense.

4. Suppose that the above facts remain correct in the infinite volume limit. QCD is always (for any $\beta$ ) in the monopole-condensed phase. Abelian charges after abelian projection are always confined and, at the same time, color charges are confined.

What about $T \neq 0$ QCD? The biggest difference from $T=0 \mathrm{QCD}$ is that the time extent is finite. Because $N_{t}$ is finite, infinitely extended monopoles can not be adopted. There is a maximum size of extendedness, so that $\beta_{c}$ is necessarily finite in contrast to the $T=0 \mathrm{QCD}$ case. We investigate what are the maximally extended monopoles and whether $\beta_{c}$ can be explained by balancing of energy and entropy of the monopole loops.

\section{The role of monopoles in the deconfine- ment transition}

It has been found that the monopole action in the confinement phase in the $T \neq 0 \mathrm{QCD}$ is the same as that given in $T=0 \mathrm{QCD}$ [7]. The energy of a monopole loop can be estimated by the self-energy term of the action. However the entropy of the monopole loops must be affected by the finiteness of the time direction. To study the entropy, we investigate the behavior of monopole loops in $T \neq 0 S U(2)$ QCD. First, we calculate the length of monopole loops [5]. Fig.1 shows the histogram of the length of monopole loops. These data are obtained from 3000 configurations in the confinement region and 1500 configurations in the deconfinement region on $16^{3} \times 4$ lattice. For $\beta<\beta_{c}(=2.298)$, a long loop exists in each 


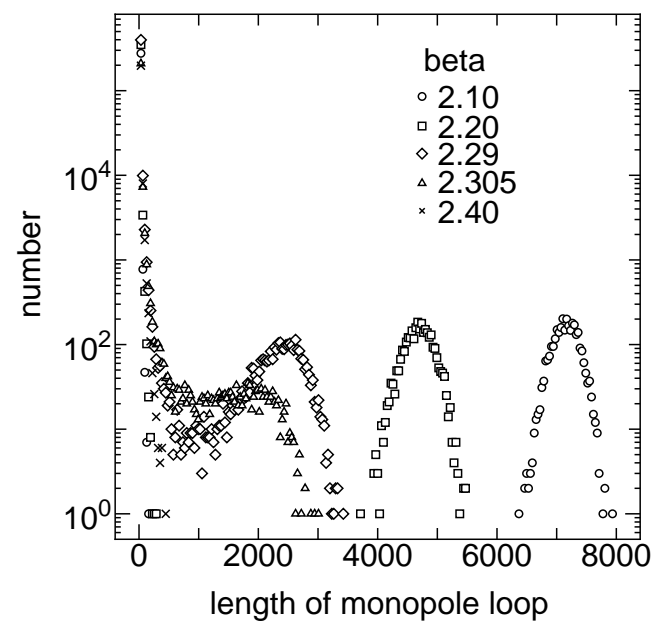

Figure 1. The histogram of the length of $1^{3}$ monopole loops on $16^{3} \times 4$ lattices.

configuration and all other loops are short. Long loops have a characteristic length at each $\beta$ and the length becomes short around $\beta_{c}$. For $\beta>\beta_{c}$, no long loop exists. In $T \neq 0 S U(2) \mathrm{QCD}$, the monopole currents contained in a long loop can reproduce the full value of the string tension [6]. This suggests that the long loop is related to the confinement mechanism in $T \neq 0$ QCD.

Next, we investigate the distribution of monopole currents contained in a long loop from its center. The mean square of the distance from the center is calculated. If the monopole currents exist uniformly in the $N_{s}^{3} \times N_{t}$ lattice, $R^{2}$ should be

$\frac{1}{N_{s}^{3} N_{t}} \int d^{4} x\left(x^{2}+y^{2}+z^{2}+t^{2}\right)=\frac{N_{s}^{2}}{4}+\frac{N_{t}^{2}}{12}$.

$R^{2}$ versus $\beta$ on $24^{3} \times 6$ lattices is shown in Fig.2. For $\beta<\beta_{c}(=2.426)$, the monopole loop is almost uniform in the whole lattice because the data are consistent with Eq.(1). For $\beta>\beta_{c}$, it is not uniform. In the confinement phase, suppose that an $n^{3}$ monopole loop spreads uniformly through the lattice, we may define the effective size of monopoles as follows:

$l^{3}(n)=\frac{\left(N_{s} / n\right)^{3} \times\left(N_{t} / n\right)}{\langle L(n)\rangle}$,

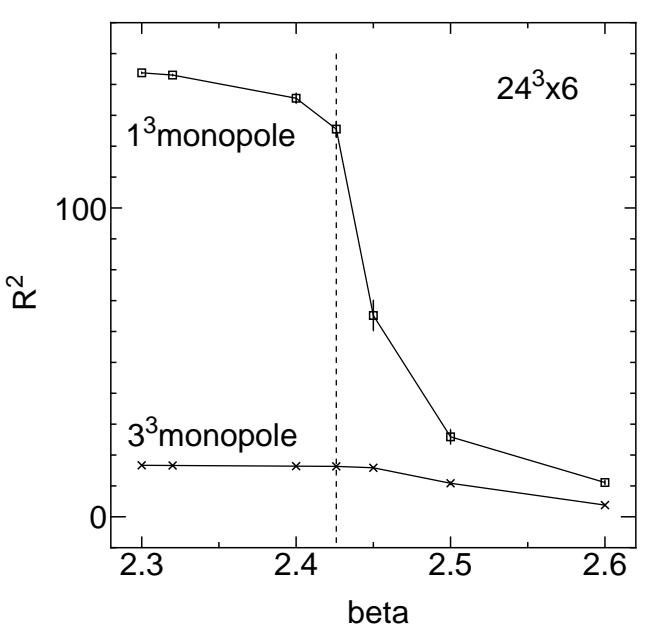

Figure 2. $\quad R^{2}$ versus $\beta$ on $24^{3} \times 6$ lattices. The dashed line indicates the location of the $\beta_{c}$.

where the numerator is the volume of the effective lattice of an $n^{3}$ monopole, which we call renormalized lattice. The denominator is the length of the monopole loop in the renormalized lattice unit. We define $\bar{l}=[l]+1$, where the symbol $[l]$ is the integer not exceeding $l$. If the monopole current with a range $\bar{l}$ is set on a link, the nearest current can be put on links $\bar{l}$ apart in the renormalized lattice unit. Hence for $n^{3}$ extended monopoles having the rounded range $\bar{l}$, the entropy can be discussed on a reduced lattice defined by

$$
\left(\frac{N_{s}}{n \bar{l}}\right)^{3} \times\left(\frac{N_{t}}{n \bar{l}}\right) \text {. }
$$

Fig. 3 shows a remarkable fact about $l(n)$ at $\beta_{c}$. $l(n)$ is always equal to the half of the time extent of the renormalized lattice $\left(N_{t} /(2 n)\right)$ at $\beta_{c}$. For example on $24^{3} \times 8$ lattice, $l(n)$ is equal to two at $\beta_{c}$ for $2^{3}$ monopole and equal to one for $4^{3}$ monopole. Then the entropy of an $n^{3}$ extended monopole loop for any $n$ should be calculated always on reduced lattices with the time extent 2 . This means that the entropy of $n^{3}$ monopole is the same for any $n$ if the original lattice is the same. What is the entropy of the monopole loop on the reduced lattice with the time extent 2 ? It is apparent that the entropy is smaller than $\ln 7$ per unit length in the $T=0$ case. The monopole 


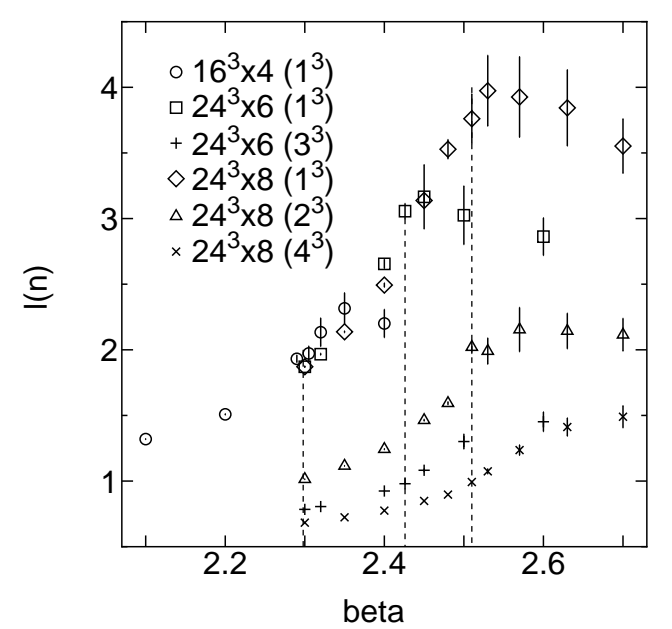

Figure 3. $l(n)$ versus $\beta$ on various lattices. The parenthesized number represents the extendedness of monopoles.

loop is very long and occupies almost all the reduced lattice sites. The time extent is only two and monopoles can not be regarded as completely point-like at $\beta_{c}$ due to their effective size. The entropy is difficult to estimate accurately. Anyway the entropy for any extended monopole on the same original lattice is the same.

On the other hand, we can see the energy per unit monopole loop length, which can be well approximated by the coupling constant of the selfenergy term of the monopole action [7]. The coupling constant is shown in Fig, We see that the energy is smaller for larger extended monopoles. To determine the deconfinement transition point, larger monopoles may be more important. What is the maximum size of extended monopole? To define the monopole currents the renormalized lattice should have two independent lattice sites in the time direction. This means the maximally extended monopole is always $\left(N_{t} / 2\right)^{3}$ one. The coupling constant of maximally extended monopoles takes about 1.16 for each original lattice at $\beta_{c}$. In Fig. $4.2^{3}\left(3^{3}, 4^{3}\right)$ monopoles are maximum for $N_{t}=4(6,8)$ and the coupling constant is about 1.16 at $\beta_{c}, 2.298(2.426,2.51)$.

In conclusion, if the entropy on a reduced lat-

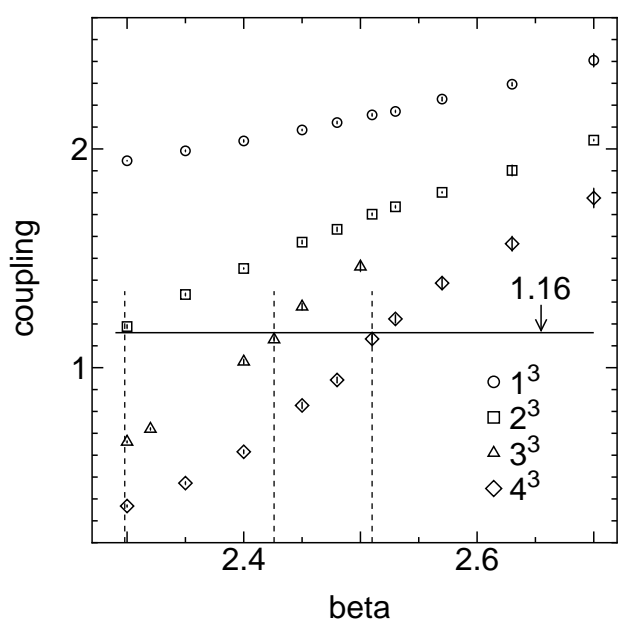

Figure 4. The coupling constant of the dominant self-energy term of the monopole action versus $\beta$ for various sized monopoles on $24^{3} \times 8$ or $24^{3} \times 6$ lattices.

tice with the time extent 2 is proved to be about 1.16, the deconfinement transition is understood by balancing of the energy and the entropy of maximally extended monopole loops on any lattices. It is interesting that the deconfinement transition may be explained by such a simple idea. We should estimate the entropy accurately to prove the statement.

\section{REFERENCES}

1. T. Suzuki, Nucl. Phys. B(Proc. Suppl.) 30, (1993) 176 and references therein.

2. G. 'tHooft, Nucl. Phys. B190, (1981) 455.

3. T.L. Ivanenko et al., Phys. Lett. 252B (1990), 631.

4. H.Shiba and T.Suzuki, Kanazawa University Report KANAZAWA 94-12, 1994.

5. A.Bode, Th.Lippert and K.Shilling, Nucl. Phys. B(Proc. Suppl.) 34, (1994) 549 .

6. S.Ejiri, S.Kitahara, Y. Matsubara and T. Suzuki, Kanazawa University Report KANAZAWA 94-14, 1994, to appear in Physics Letters B.

7. S.Kitahara, Y.Matsubara and T.Suzuki, in preparation. 